\title{
Using simulated patients in medical and health professions education
}

\author{
Albena Gayef ${ }^{1}$ \\ Trakya University Faculty of Medicine, Department of Medical Education, Edirne, TURKEY
}

\begin{abstract}
Interactive methods, which are one of the effective training methods used in medical and health professions education enable students to participate actively in the learning process. In this context, simulated patients can be used in simulation applications which are interactive methods. The simulated patient is an educated person to revive and present the real patient scenario and offers a safe learning opportunity based on experience in a virtual or laboratory environment prior to the real patient environment. This application enables to students to increase knowledge and skill level, self-efficacy, motivation and selfconfidence, improves clinical decision making and communication skills. The aim of this study is to review simulated patient applications in medicine and health sciences education and to provide a detailed overview on using simulated patients for both teaching and assessment purposes in medical and healthcare professional education.
\end{abstract}

\section{Introduction}

Policy documents on medical education from bodies such as the United Kingdom's General Medical Council and the Association of American Medical Schools recommend that medical schools ensure (early) patient contact. Simulated patients are a valuable resource for teaching and assessing communication and clinical/ physical examination skills in medicine [1]. Simulated patients are now used not only in medicine but across the range of healthcare professional education and training including nursing, dentistry, physiotherapy [2], dietetics [3] and pharmacy [4]. All simulated patients play roles, they simulate 'real' patients. Simulated patients can also be used to give feedback to students and evaluate performance. To use simulated patients effectively, resources and staff time must be dedicated to recruiting, training and managing an simulated patients 'bank'. The introduction of early clinical practice in the undergraduate medical curriculum has lead to a need for more patient participation in teaching and learning. However, at the same time, the availability of patients for teaching and learning medicine has been influenced by changes in healthcare delivery. A reduction of inpatient beds and a shift to care in the community and reduced average hospital admission period for patients has had a major impact on the availability of patients to take part in the training of healthcare professionals [1].

\section{Simulated patients}

There are various ways to recruit simulated patients. If you are starting small, to perhaps pilot an simulated patients programme, asking colleagues and local community contacts can suffice as a method of recruiting simulated patients. You can recruit from the general public by placing advertising posters or leaving brochures at strategic places such as hospital and general practice waiting rooms, or community sites such as churches, student organization buildings or resource centres [1].

Simulated patients can be used for teaching and assessment purposes. Teaching simulated patients can be used to train students in the consultation skills and communication skills in general [5].

The aim of using simulated patients is to simulate the range of skills and topics involved in real consultations. Students interact with simulated patients as though they were taking a history, carrying out an examination or giving information to a real patient. Many different scenarios or roles are needed if simulated patients are used throughout the curriculum. These will range from straightforward history taking scenarios for preliminary consultation skills training with relatively inexperienced students to, for example, complex breaking bad news and psychiatric scenarios for students and doctors further on in their training. An simulated patient role may cover part of the consultation (e.g., giving a history) to all components of a full consultation including physical examination (see below), and asking questions about treatment and

${ }^{1}$ Corresponding Author: albenag77@gmail.com 
management plans. Physical examination and procedural skills. In terms of physical examination, where the purpose of teaching (or assessment - see below) is to assess the technique of physical examination or procedural skills, an simulated patient with normal signs can be used for teaching and learning. If the purpose is to measure a student's ability to identify important physical signs then real patients with these signs will usually be required. In performing the simulation, the simulated patient presents the gestalt of the patient being simulated; not just the history, but the body language, the physical findings, and the emotional and personality characteristics as well [1].

There are different methods and techniques may be used for communication skills training in medical education and health professions education. Simulated patient practice is used as a high level of reliability and prevalence as a training and evaluation tool that was initiated by Howard Barrows and Steve Abrahamson in the 1960s [6,7]. The simulated patient consists of people who accurately and consistently mimic the condition of patients and participate in clinical and communication skills training $[8,9,10]$. Simulated patient practice has an important role as an effective learning tool in medical education and health sciences education for gaining basic communication skills. Communication skills are very important in medical education and health professions education. A quality communication with a patient / healthy person is indispensable in achieving health and maintaining health. Communication skills include being optimistic and understanding, empathic to the patient's situation, showing interest, helping the patient to express and deal with feelings of anxiety and the like, and building a secure relationship with the patient. In addition, questions such as asking questions, giving information, dealing with psychosocial problems, smile, touching, establishing eye contact, using body language, active listening, understanding the patient and not being judgmental are important elements in communication skills $[11,12]$. Simulated patients are now used widely for teaching and assessment purposes in medical and healthcare professional education. Simulated patient application consists of three stages. These are: pre-information, application and analysis. In the preliminary information which is the first stage, students are informed about simulated patient practice, expected goals and objectives. During the application, students are interviewed with simulated patients. In the final stage, students can share their learning and skill experiences in a non-judgmental atmosphere and have the opportunity to express their communication techniques, their experiences and thoughts in practice $[2,6]$.

\section{Advantages and Disadvantages of using Simulated patients}

While the introduction of simulated patients occurred for the reasons, it is worth stating early on that simulated patients have many more advantages than just assessment reliability compared to real patients. They are available as and when required. They can be trained in a broad range of clinical cases, thus giving students a variety of experiences that they may not encounter in real patients. They are willing and ready to undergo scenarios many times. Their behaviour is predictable. They can be used in situations where the use of a real patient would be inappropriate (e.g., practising giving a terminal diagnosis). The main disadvantage of using simulated patients is the cost: it involves dedicated staff and financial resources. The other main disadvantage of using simulated patients is that they are not 'real': however, it is worth reassuring less enthusiastic colleagues that studies indicate that much research shows that welltrained simulated patients are not usually distinguishable from real patients [1].

\section{Conclusion and Recommendations}

Simulated patients have been used in teaching and assessment in medical and health professions education training for 40 years. Their use in medical education is now worldwide. There are many advantages of using simulated patients perhaps most particularly in standardizing teaching and assessment so all students have the same experience. Recruiting, training and using simulated patients requires expertise and ongoing resources. Simulated patient performance requires ongoing monitoring and assessment, both in terms of validity and reliability, and in terms of the impact on the simulated patient his or herself [1].

\section{References}

1. J.A. Cleland, K. Abe, J.J.Rethans, The use of simulated patients in medical education: AMEE Guide No 42, Medical Teacher, 2009; 31: 477-486 (2009).

2. C. Lane, S. Rollnick, The use of simulated patients and role-play in communication skills training: a review of the literature to August 2005. Patient Education and Counseling 67 13-20 (2007).

3. D. Beshgetoor, D. Wade. Use of actors as simulated patients in nutritional counseling. J Nutr Educ Behav. 39 (2):101102 (2007).

4. M.C. Watson, P. Norris, A.G. Granas, A systematic review of the use of simulated patients and pharmacy practice research, The International Journal of Pharmacy Practice, 14: 83-93. June (2006).

5. Kinnersley \& Pill. Potential of using simulated patients to study the performance of general practitioners, British Journal of General Practice, 43, 297-300 (1993).

6. L.D. Howley, J. Martindale. The efficacy of standardized patient feedback in clinical teaching. a mixed methods analysis. Med Educ Online, 9:4356 (2004). 
7. P. Michael, E. Pagano, S.H. Campbell, L.M. Currie, E. Chamberlim, C.A. Pates. Validating the health communication assessment tool (HCAT). Clin Simul Nurs, 11:402-410 (2015).

8. J.M. Eagles, S.A. Calder, S. Wilson, J.M. Murdoch, P.D. Sclare. Simulated patients in undergraduate education in psychiatry. Psychiatr Bull, 31:187-190 (2007).

9. C.A. Ryan, N. Walshe, R. Gaffney, A. Shanks, L. Burgoyne, C.M. Wiskin. Using standardized patients to assess communication skills in medical and nursing student. BMC Med Educ, 10:24 (2010).

10. S. Turan, S. Üner, M. Elçin. The impact of standardized patient feedback on student motivational levels. Balkan Med J, 28:43-48 (2011).

11. I.P. Kruijver, A. Kerkstra, J.J. Kerssens, C.C. HoItkamp, J.M. Bensing, H.B. Wiel Communication between nurses and simulated patients with cancer: evaluation of a communication training programme. Eur J Oncol Nurs, 5:140150. (2001).

12. L.L. Hsu, W.H. Chang, S.I. Hsieh. The effects of scenario-based simulation course training on nurses' communication competence and self-efficacy: a randomized controlled trial. J Prof Nurs, 31:37-49 (2015). 